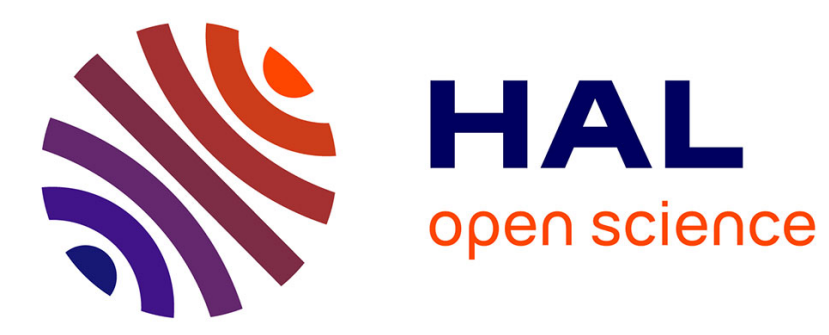

\title{
Modeling of the influence of the defect position on the reflected intensity in EUV masks
}

\author{
Maxime Besacier, Patrick Schiavone, Vincent Farys, Rafik Smaali
}

\section{To cite this version:}

Maxime Besacier, Patrick Schiavone, Vincent Farys, Rafik Smaali. Modeling of the influence of the defect position on the reflected intensity in EUV masks. 2005, pp.629-639, 10.1117/12.598415 . hal00023203

\section{HAL Id: hal-00023203 \\ https://hal.science/hal-00023203}

Submitted on 21 Apr 2006

HAL is a multi-disciplinary open access archive for the deposit and dissemination of scientific research documents, whether they are published or not. The documents may come from teaching and research institutions in France or abroad, or from public or private research centers.
L'archive ouverte pluridisciplinaire HAL, est destinée au dépôt et à la diffusion de documents scientifiques de niveau recherche, publiés ou non, émanant des établissements d'enseignement et de recherche français ou étrangers, des laboratoires publics ou privés. 


\title{
Modeling of the influence of the defect position on the reflected intensity in EUV mask
}

\author{
Maxime Besacier, Patrick Schiavone, Vincent Farys, Rafik Smaali \\ Laboratoire des Technologies de la Microélectronique CNRS, c/o CEA Grenoble, 17 Avenue des \\ Martyrs, 38054 Grenoble Cedex 09, France
}

\begin{abstract}
In Extreme Ultraviolet Lithography, the electromagnetic modeling of the mask allows to determine the influence of the mask structure on the electromagnetic field. That makes it possible to take into account the presence of a defect modifying the multi-layer stack [1][2]. This paper presents the results of simulations, performed using a modal method, on the aerial image of the reflected intensity above the resist depending on the position of a defect with respect to an absorber pattern. These simulations allow to consider the influence of a defect not only on top of the structure but also everywhere inside the multilayer. The current method is the MMFE: Modal Method by Fourier Expansion. Modal methods are well adapted for EUV simulation mask due to materials and structure size.
\end{abstract}

Keywords: EUV Lithography, simulation, defective mask, modal method, absorber pattern, aerial image, planarization effect.

\section{INTRODUCTION}

Compared to other classical optical techniques, EUV lithography requires reflective optics. The EUV mask is a Bragg mirror. An absorber material (for instance Chromium) is deposited on a stack of $40 \mathrm{Mo} / \mathrm{Si}$ bilayers and the presence of a defect inside or under the multi-layer stack is one of the most severe technical issue that have to be overcome to make the technology available. This process is still not very developed so modeling and simulation are very important to understand and to improve this new technique of lithography. An electromagnetic approach has to be used to represent what happens after reflection on the mask but also inside the mask structure. This type of simulation must allow to take into account defects inside the multi-layer. The problem of defect inside the multi-layer has already been addressed by several authors [1-5] and some of them have shown that a perturbation inside the multi-layer stack, even if it is not propagated up to the mask surface, can disturb the electromagnetic field and so the projected image on the wafer.

In this paper, using electromagnetic modeling of the light field inside and above the mask, we study the influence of the defect position with respect to an absorber pattern.

The modelling method is the MMFE (Modal Method by Fourier Expansion). We will show that the MMFE is well suited to model problems where the wavelength is short compared to the feature size. This method allows to address, with efficiency, modelling of topography with both sharp (absorber) and smooth (defect) shapes. This study is carried out in two dimensions.

In the first part, after a general description of the MMFE, we present the modelling method to consider the defect propagation through the stack. The propagation models take into account a smoothing or a growth of defect with respect to the deposition process. Even if it could be not really physically realistic, it allows to consider the disturbance effect of each layer inside the stack and especially on surface of the mirror. To improve and to complete this kind of study, measurements have been done with programmed defects to know the topography at the top surface with respect to the initial defect shape [6-9]. Moreover, P.Naulleau [10] has shown with printing results that smoothing-optimised coatings reduce significantly the printability of substrate defects. We have chosen to present both gaussian and circular shapes, but any type of defect shape could also be considered in our simulations.

In the second part, we remind the impact on the reflected electrical field of a defect in a blank structure. This allows to show that the size but also the deposition conditions can have a significant effect on the reflected field.

In the last part we present the impact of a defect in a EUV mask structure, that is with an absorber layer above the multilayer. The main interest is to observe the impact of a defect in the vicinity of the absorber considering the horizontal position and the feature size. 


\section{DESCRIPTION OF MODELING}

\subsection{Rigorous method MMFE}

A full description of this method can be found elsewhere [11] and will not be detailed here. Only a brief description is given. The Modal Method by Fourier Expansion is well adapted for the topography of the mask in EUV lithography. MMFE is based on a simple approach where Maxwell equations are solved thanks to a matrix system. TE or TM polarization are treated using the same formalism and an improved version allows to obtain good results for TM polarization in terms of convergence speed. In our study only the TE polarization has been addressed. This method implicitly considers periodic structures. The solution of Maxwell equations is reduced to the solution of an algebraic eigenvalue problem in a discrete Fourier space. The derivation of the matrix operator involves two steps:

- the electromagnetic field is expanded into Floquet-Fourier series,

- the permittivity of the materials which appears in the Maxwell equation is here a periodic function and is expanded into Fourier series.

The topography of the structure which is studied is cut in different layers. These different layers are linked by a $\mathrm{S}$ matrix system which accounts for the boundary condition providing a good convergence even for thick or absorbing layers. In this rigorous method, the only approximation is due to the number of modes retained for the truncation of the modal expansion. In EUV lithography, due to the short wavelength, the ratio $\frac{\lambda}{d}$ is very small. Consequently the number of mode is important according to the following equation:

$$
\sin \left(\theta_{m}\right)=\sin \left(\theta_{0}\right)-m \cdot \frac{\lambda}{d}
$$

$\theta_{0}$ is the incident angle

$\mathrm{m}$ is the number of mode

$\theta_{\mathrm{m}}$ is the angle of the mode $\mathrm{m}$.

This large number of mode leads to a compromise between computation time and results accuracy. In practice, to address complex topographies we have simulated in this article (defective multi-layer structure with an absorber layer) and for a retained number of mode of 51, the precision is good and the computation of the reflected field is performed in around 20 seconds with an Intel Xeon $3.4 \mathrm{GHz}$ processor.

This method allows to obtain the field above the mask but also inside the structure. The reflected field above the mask is an interesting data because this is the first data which can provide us an estimation of the pattern size printed in the resist. But this result is not the ultimate step, it is also necessary to know the field characteristic at the wafer (Aerial Image), after passing through the projection lens. Our code, which computes the reflected field at the mask, is then coupled with a lithographic commercial software (SOLID-C), in order to obtain the aerial image.

\subsection{Model of defect shape}

The real shape of the defect is difficult to model properly, moreover it appears, in this kind of modeling, that this feature is not the dominating one. Especially because the multilayer deposition on the defect leads to a planarization or a growth effect of this defect. This paper is not focused on a realistic deposition process modeling. Most of the time, the defect is defined as a sphere or a cylinder for 2D structures [6]. In this paper we decided to address problems with circular and gaussian shape, but any other shapes can be handled in our models.

\subsubsection{Circular defects}

These defects are modeled with the general parametric equations of an ellipse:

$\mathrm{x}(\mathrm{t})=\mathrm{a} \cdot \cos (\mathrm{t})$

$y(t)=b \cdot \sin (t)+b$

$\mathrm{a}$ and $\mathrm{b}$ are respectively the half lengths of the ellipse in the $\mathrm{x}$ direction (parallel to the surface) and in the $\mathrm{y}$ direction (orthogonal to the surface). The parameter $t$ is defined between $-\pi / 2$ and $\pi / 2$. To address a circular defect, the parameter a must be equal to the parameter $b$. In most of studies presented below, we address a circular shape for the defect. Our studies are in two dimensions, consequently the defect is considered as a infinitely long line in the $\mathrm{z}$ direction.

P.B.Mirkarimi [6] has shown that in both cases of near-normal and off-normal deposition incidence on the height of defect on surface of the multi-layer is reduced with respect to the initial perturbation. In order to consider this 
compression, the propagation of the defect through the multi-layer is modelled thanks to the parametric equations of the parallel curves [12] where a compression coefficient $\alpha$ is inserted as follow:

$$
\left\{\begin{array}{c}
x p(t)=x(t)+d \frac{y^{\prime}(t)}{\sqrt{x^{\prime}(t)^{2}+y^{\prime}(t)^{2}}} \\
y p(t)=y(t)-\alpha \cdot d \frac{x^{\prime}(t)}{\sqrt{x^{\prime}(t)^{2}+y^{\prime}(t)^{2}}}
\end{array}\right.
$$

The coefficient $\alpha$ allows us to study different types of deposition processes. $\alpha=1$ means the defect is propagated without planarization effect. The curves at different stages are parallel and finally on surface of the multi-layer, even if the width of defect is different, the height is still the same than the initial one. $\alpha>1$ can be interpreted as a planarization effect and on the opposite $\alpha<1$ can be interpreted as a growth of the defect size with respect to the initial one.

The parameter $\mathrm{d}$ is the distance between the initial surface of the defect and the y position where the profile is drawn $x^{\prime}(t)$ and $y^{\prime}(t)$ are the derivatives of $x(t)$ and $y(t)$ respectively. Three examples of planarization effects are drawn in Figure 1.

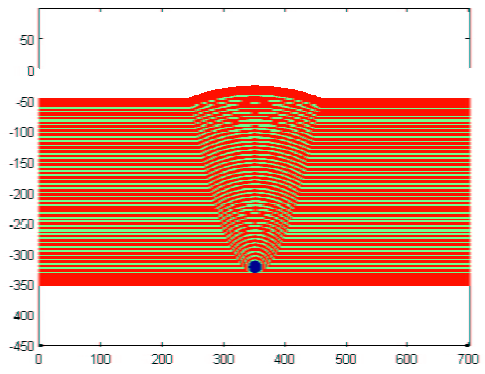

a) $\alpha=1$

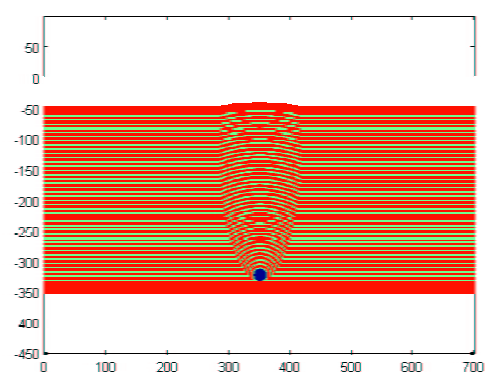

b) $\alpha=1.05$

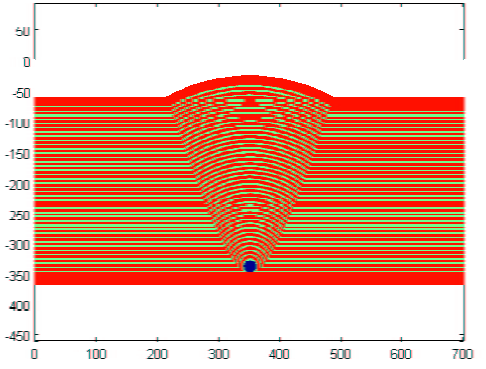

c) $\alpha=0.95$

Figure 1: $\alpha$ parameter impact on the deposition of the multi-layer. 20nm diameter circular defect.

\subsubsection{Gaussian defects}

To represent this shape of defect we do not use, as the previous circular defect shape, parametric equations. Indeed, a gaussian shape is a function $\mathrm{y}=\mathrm{f}(\mathrm{x})$. The formula of a gaussian shape is defined with the well known equation:

$y=h \cdot \exp \left(-\frac{x^{2}}{2 \cdot \omega^{2}}\right)+d \quad$ (for the first layer, $\mathrm{d}=0$ )

$\mathrm{h}$ is the size of the defect.

$\mathrm{d}$ is the distance between the initial surface of the defect and the $\mathrm{y}$ axis where the profile is drawn.

To model this type of defect shape, we have investigated two ways which allow to address the deposition effect on the propagation of the defect. In the first approach, we have inserted a coefficient which can be interpreted as the planarization effect. It appears on the the parameter according to the following equation:

$\omega=\frac{F W H M}{2 \sqrt{2 \cdot \log 2}}+c . d$

FWHM is the Full Width Half Maximum parameter.

$\mathrm{c}$, in the same way than the parameter $\alpha$ for the circular defect, can be expressed as a compression or a planarization coefficient.

Results on defect shape with respect to this c parameter are presented below in figure 2. 


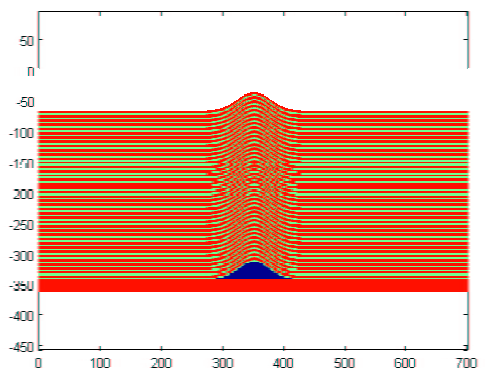

a) $\mathrm{c}=0$

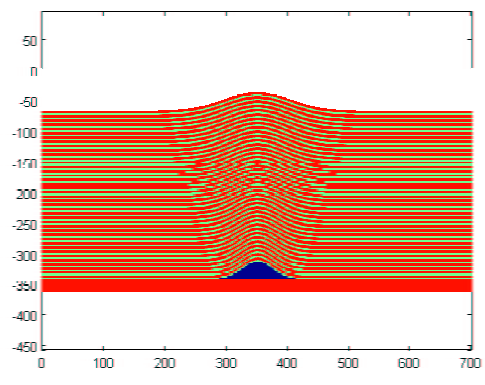

b) $\mathrm{c}=0.1$

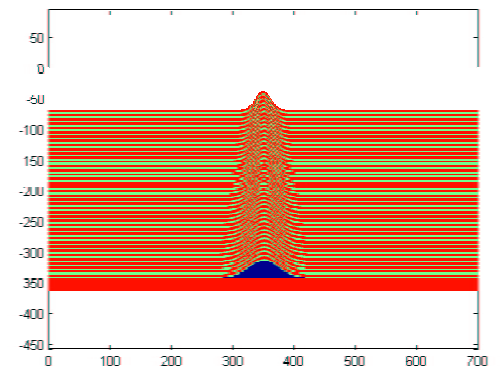

c) $\mathrm{c}=-0.05$

Figure 2: c parameter impact on the deposition of the multi-layer. Defect size: h=30nm, FWHM: 60nm

This approach, as for the circular defect does not allow to manage precisely the size of the defect at top of the multilayer. However, this parameter is interesting to keep because this is a data which can be known thanks to experiments. So this modeling method is not the best way to take into account the modification of the surface topography induced by the defect.

The second approach consist in defining two gaussian shapes: first for the initial defect and a second to represent its impact on surface. This way of modeling is better in view of comparison with measurements but certainly not much more realistic. The topography of a layer at a distance $d$ with respect to the initial defect is computed thanks to the following linear approximation:

$$
y_{d}=y_{b}+\frac{\left(y_{t}-y_{b}\right) \times d}{h_{l}}+d
$$

where:

$\mathrm{h}_{\mathrm{l}}$ is the height of the complete multi-layer.

$\mathrm{y}_{\mathrm{t}}$ and $\mathrm{y}_{\mathrm{b}}$ are respectively gaussian shapes of the defect on top and on bottom of multi-layer. Both analytical equations are expressed with the following equations and the parameter definition is the same than in previous approach (with $\mathrm{c}$ parameter equal to 0 ).

$y_{t}=h_{t} \cdot \exp \left(-\frac{x^{2}}{2 . \omega_{t}^{2}}\right)$

The figure presented below (Figure 3 ) is an example for the configuration:

Defect shape: $h_{b}=30 \mathrm{~nm}$

$$
\mathrm{FWHM}_{\mathrm{b}}=60 \mathrm{~nm}
$$

Surface shape: $h_{t}=15 \mathrm{~nm}$

$\mathrm{FWHM}_{\mathrm{t}}=80 \mathrm{~nm}$

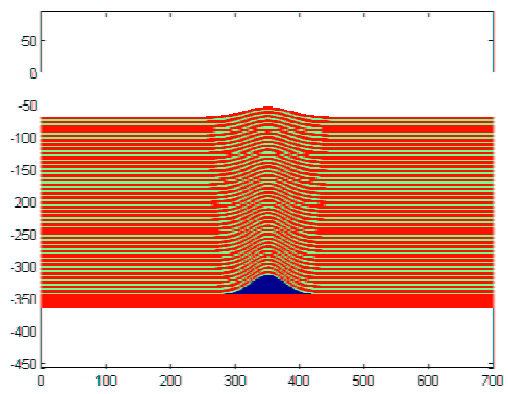

Figure 3: modeling of the deposition impact for a gaussian description of the defect shape and the surface topography.

Note that, the multi-layer structure will be identically if we chose the first approach with the coefficient equal to 0 or the second approach with the same shape of defect on top and on bottom. 


\section{IMPACT OF DEFECTS ON THE REFLECTED INTENSITY}

This third part focuses only on the influence of a defect within a multi-layer with no absorber stack deposited on it. Some results have been already presented by P.Schiavone [11].

A study of the defect shape impact on the perturbation of the reflected intensity is difficult to carry out because the main parameter of the defect propagation through the multi-layer is still the deposition process. A pertinent analysis consists in considering one defect shape and studying the aerial image according to the defect size for one deposition process, and also by the other side, the aerial image according to the deposition process for one defect size.

In every simulations that we have done and for the results presented in this paper, several parameters remain constant: Wavelength $13.5 \mathrm{~nm}$, incidence angle $5^{\circ}$, the multi-layer is made of 40 pairs of Mo $(2.74 \mathrm{~nm}$ thickness $) / \mathrm{Si}(4.11 \mathrm{~nm})$ and the defect material is chromium. For aerial image computation, the reduction ratio is 4 , the partial coherence is 0.7 and the numerical aperture is 0.25 .

The following simulation results are performed with a circular defect shape with a diameter ranging from 10 to $100 \mathrm{~nm}$. For example, aerial images are addressed for three planarization coefficient values (figure 4).

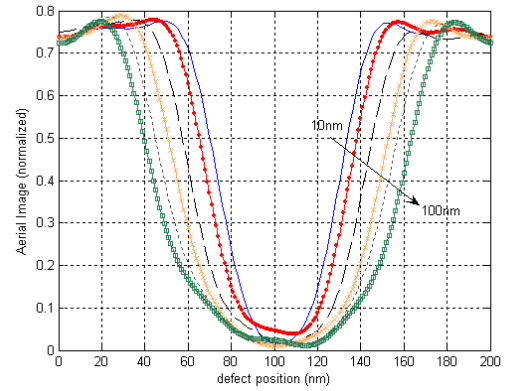

a) $\alpha=0.96$

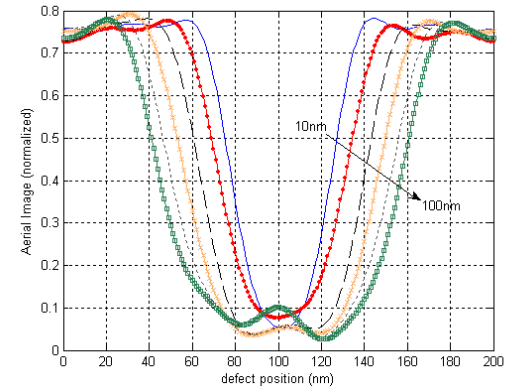

b) $\alpha=1$

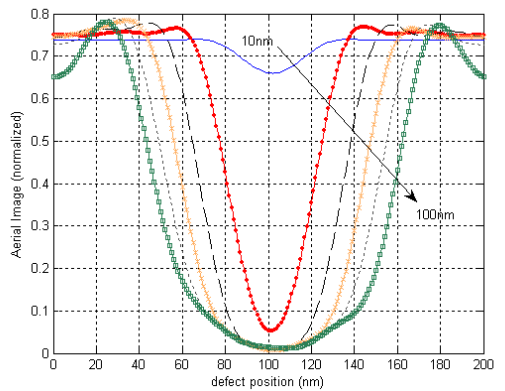

c) $\alpha=1.06$

Figure 4: Aerial image for different defect sizes and 3 different planarization deposition conditions.

These pictures show that the deposition process (characterized here by the $\alpha$ coefficient) has a major impact on the influence of the defect in the aerial image. A large planarization effect (Figure 4c) is important in order to reduce the drop of intensity or the width of the aerial image due to the presence of a defect. It is clearly represented for the small defect sizes with a comparison between figure $4 \mathrm{a}$ and figure $4 \mathrm{~b}$. Nevertheless for $\alpha=1$ (figure $4 \mathrm{~b}$ ), the intensity curve shows a bump, in particular for big defects. It can be explained by the fact that for large size defects there is a flat at this position [13]. And especially, for this coefficient value, the periodicity of the multi-layer stack is not disturbed. This assumption is confirmed with figures $4 \mathrm{a} \& 4 \mathrm{c}$, where, in spite of the big defect sizes, no bumps are observed, because of the change of periodicity.

The printed CD versus the defect size for different coefficient values is summarized on figure 5 .

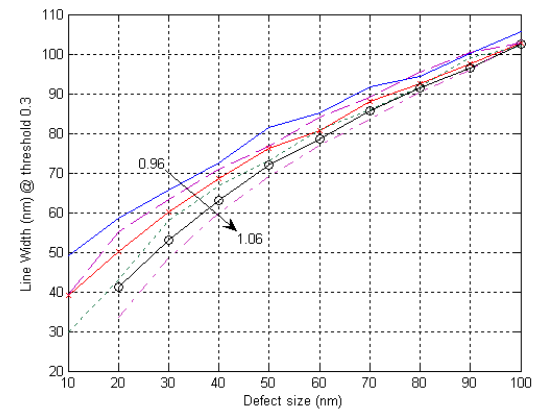

Figure 5: Printed CD versus Defect size for a range of planarization coefficient from 0.96 to 1.06

With no surprise, the printed CD increases with the size of the defect. This CD is larger when the planarization coefficient is small. This difference of CD versus planarization coefficient is less important for big defects compared to the small ones. It can be also noticed that a coefficient higher than 1.04 and for a defect smaller than 20nm, the drop of intensity is not sufficient to print. 
For low defects, the effects of the planarization minimize the impact of the defect.

\section{INFLUENCE OF DEFECT WITH RESPECT TO THE ABSORBER}

Considering only the defect influence on a blank does not allow to address the full problem in the case of a EUV mask. Indeed, the defect position as the size with respect to the absorber features can have a non negligible influence on the reflected intensity of the near electrical field and then on the aerial image [13]. In this part we will study first the impact of the defect in the vicinity of an absorber pattern and the way it affects the aerial image. Secondly we will look at the influence of the absorber size when it is deposited at the same position (horizontal coordinates) than the defect. And finally we will look at the effect caused by the change of the defect size under an absorber pattern. Note that for these simulations, general parameters remain the same than those defined in the previous section.

\subsection{Influence of the defect position with respect to the absorber}

To address this problem, different configurations (several are shown below) have been modeled. The absorber pattern is made on a buffer layer (15nm thickness) in $\mathrm{SiO} 2$ and a chromium layer ( $85 \mathrm{~nm}$ thickness). The width is $120 \mathrm{~nm}$. The defect has a gaussian shape (20nm height and 20nm FWHM). The propagation of this shape through the multi-layer induces at the mirror surface a gaussian deformation (20nm height and 40nm FHWM). Different topographies are shown on the figure 6 .
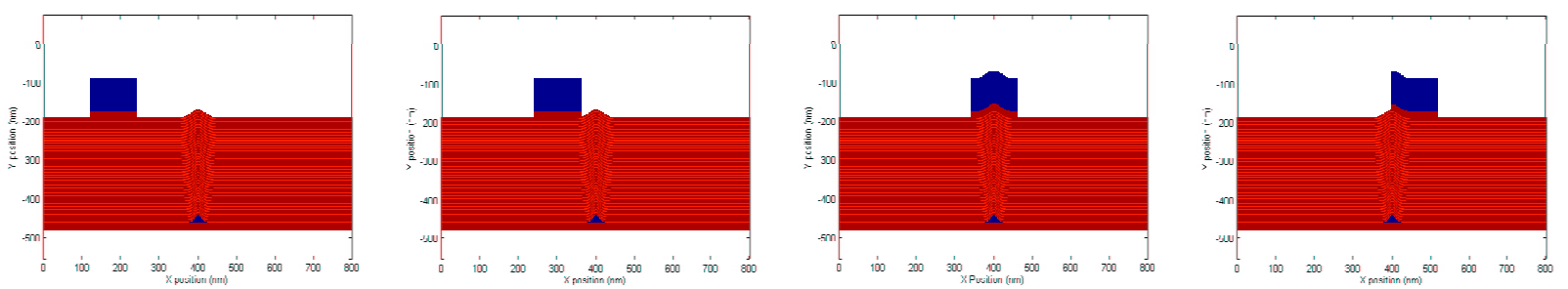

Figure $6: 4$ different representation of a 2D EUV mask with defective structure.

The simulation results, shown in figure 7, give the aerial images of the geometries shown in figure 6 . These curves show a comparison between a defective mask structure and a mask with no defect.

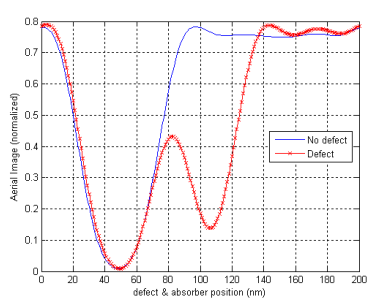

a)

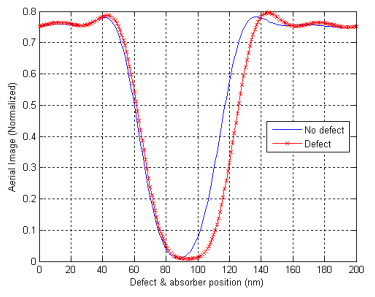

b)

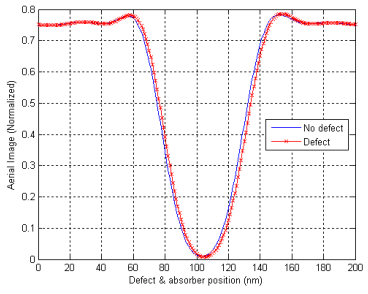

c)

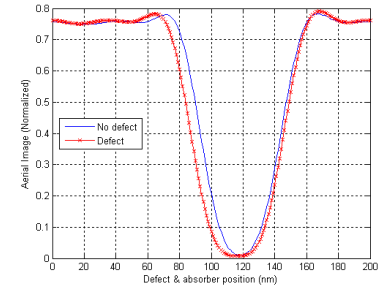

d)

Figure 7 : Aerial image for the 4 topographies defined in figure 6, with and without defect.

When the absorber pattern is far from the defect position (case a), two different drops of intensity appear which can lead to a double print, one for the absorber pattern and one for the defect. This is not the case anymore when the absorber is close to the defect. The drop of intensity is larger compared to the aerial image with no defect (case b \& d). This difference between ideal and defective topographies is reduced when the absorber covers the defect. The printed CD (for the absorber) versus the absorber position can be drawn (figure 8): 


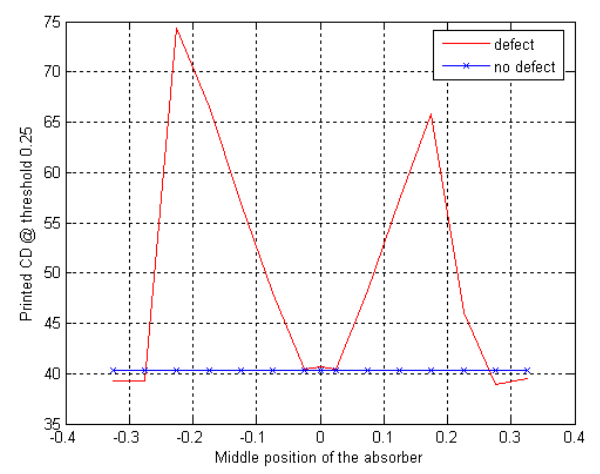

Figure 8 : Printed CD versus absorber position.

Note that in the horizontal scale the middle position corresponds to the center of the absorber. This scale is normalized, it means that the position of the center at 0 corresponds to an absorber position just above the defect. The blue curve is the printed $\mathrm{CD}$ of the absorber with no defect, it is constant whatever the position of the pattern.

The different simulation configurations have respected a symmetry of the absorber position on both sides of the defect. Nevertheless, we do not observe this symmetry on the printed CD. The main reason is the shadowing effect of the absorber. This shadowing effect, that we have studied in previous work [14], is mainly due to the angle of incident light (figure 9).

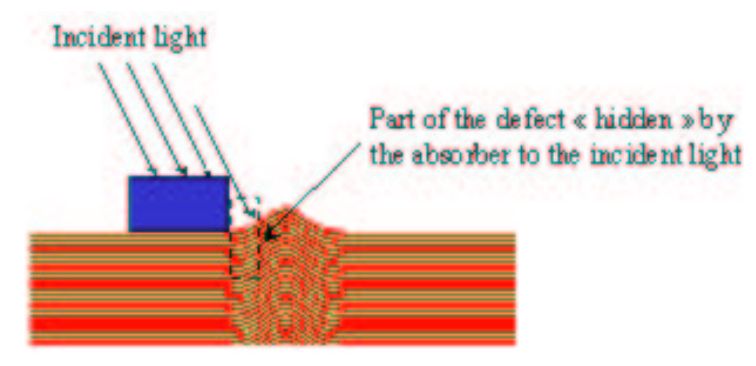

a) Absorber on the left side of the defect

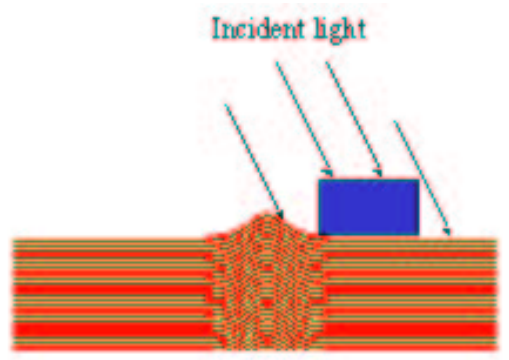

b) Absorber on the right side of the defect

Figure 9: Schematic shadowing effect representation on a defective mask surface.

If the absorber is close to the defect on the left side (figure 9a), a part of defect influence will be "hidden" by the thickness of the absorber and then will not have influence on the printed CD. The symmetric position (figure $9 \mathrm{~b}$ ) will not lead to the same effect. Indeed, in this case, the full defect will be enlightened. So for two symmetric positions, an absorber on the right side of the defect will lead to a larger printed CD than an absorber on the left side. This effect remains available while both drops of intensity due to defect and absorber are near and lead to one drop.

\subsection{Influence of the absorber size.}

Results presented in the previous part has shown that when the absorber covers the deformation at the top of the multilayer caused by the defect, the printed CD matches well with the CD computed with no defect. The question is: "Is it always true whatever the size of the absorber or the defect ?"

In this part we will look at the impact of the absorber thickness which ranges from 10 to $105 \mathrm{~nm}$. The width is constant and equal to $120 \mathrm{~nm}$. The capping layer thickness remains constant at $15 \mathrm{~nm}$. Two configurations are modeled: The first with the defect fully covered by the absorber (figure 9a) and the second one with a perturbation of the top surface larger than the absorber width (figure $9 b$ ).

Note that the x positions of both center of the defect and of the absorber are still the same. The defect lies just underneath the absorber layer. 


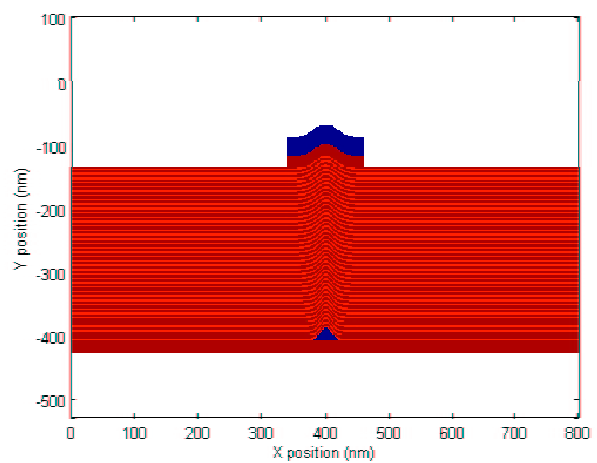

a) defect size:h=20nm, FWHM=20nm

At top of surface: $\mathrm{h}=20 \mathrm{~nm}, \mathrm{FWHM}=40 \mathrm{~nm}$

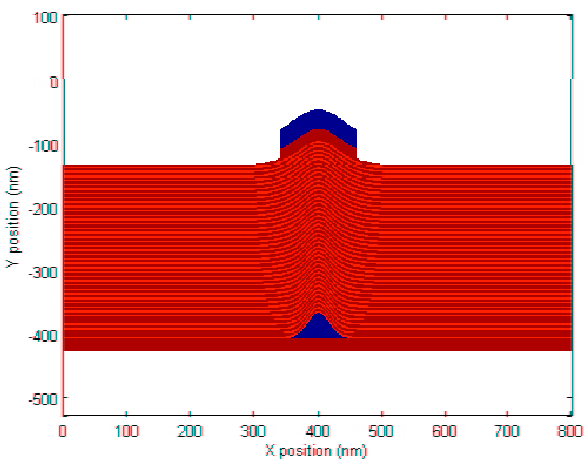

b) defect size:h=40nm, FWHM=40nm

At top of surface: $h=40 \mathrm{~nm}, \mathrm{FWHM}=80 \mathrm{~nm}$

Figure 10: simulation configurations : defect fully covered (a), and partially covered (b).

For instance, three results are shown in figure 11. It compares for three different absorber thicknesses $(10,50 \& 100 \mathrm{~nm})$ the aerial image for a topography with no defect, a topography with a small defect, it means fully covered, and a large defect, partially covered.

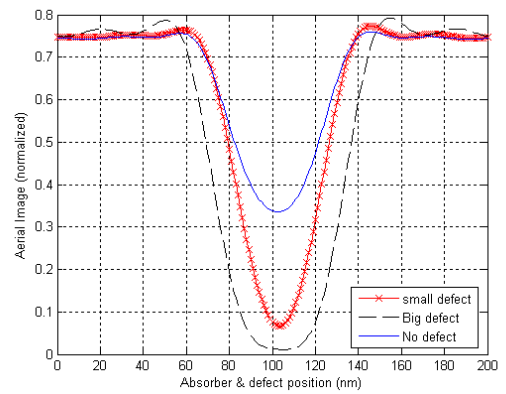

a) absorber thickness : 10nm

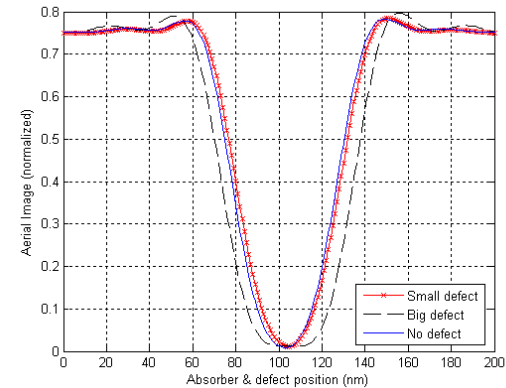

b) absorber thickness : $50 \mathrm{~nm}$

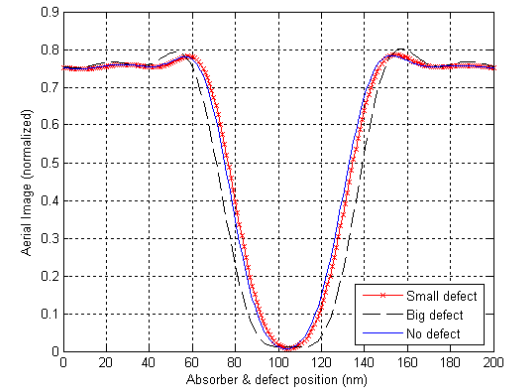

c) absorber thickness : 100nm

Figure 11: Aerial image for three thicknesses and for three different topographies.

For low absorber thickness, even if the defect is fully covered by the absorber layer, it can have an effect on the aerial image. It is translated into a deeper drop of the light intensity. This effect does not seem to be so critical, because it strengthens the main effect of the absorber, that is the drop of intensity. For thicker absorber layers, the impact of a defect is hidden for small size defect and the two aerial images with small defect and with no defect match well. For large defects the aerial image is not dependant of the absorber thickness. We can conclude, thanks to the figure 12 that for small defect sizes compared to the absorber width, the printed CD is the same than the one obtained with no defect, except for small absorber thickness. For large defect size, whatever the thickness of absorber, the aerial image remains almost constant. The aerial image and consequently the printed CD only depends on the defect size. That is also what we will observe in the last part. 


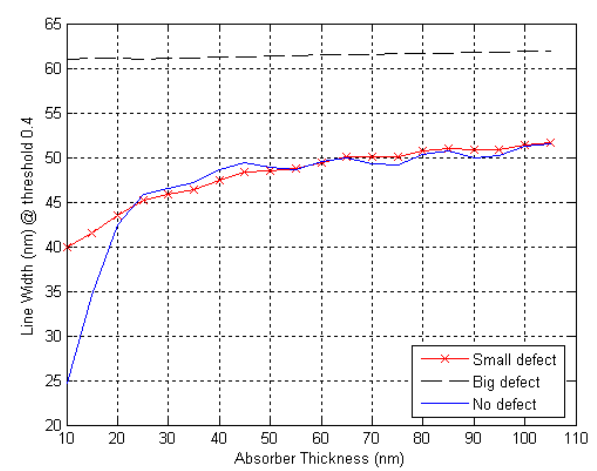

Figure $12:$ Printed CD versus absorber thickness

Small oscillations on the blue curve are typical of the type of interference effects between light reflected from the top of the absorber and light reflected off the multi-layer mirror that are described in [15] for the line width. On the red curve these oscillations still exists but their amplitude is less important. It is not visible anymore on the dotted black curve. This drop of amplitude in oscillations are probably due to the deformation of the top surface of the absorber due to the defect presence.

\subsection{Influence of the defect size}

This last part shows the printed CD computed with a constant absorber size (thickness 100nm and width $120 \mathrm{~nm}$ ) and for different defect sizes ranging from 1 to $100 \mathrm{~nm}$. Remember that the defect shape is a gaussian. We have defined that a defect with a size at $10 \mathrm{~nm}$ for instance, corresponds to a gaussian with an height and a FWHM equal to $10 \mathrm{~nm}$. The deformation shape at top of multi-layer surface will be a gaussian with the same height and a FWHM parameter twice bigger. For this range of sizes, the printed CD is compared to printed CDs computed with the absorber only and with the defect only. Results are shown below (figure 13).

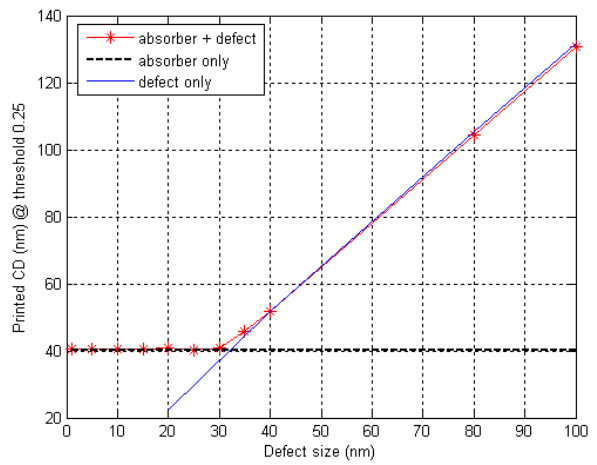

Figure 13 : Printed CD versus the gaussian defect size.

The mask structure with an absorber layer deposited on a non defective multi-layer leads to a printed CD represented with the black dotted line. The blue curve represents the CD computed with a defective multi-layer with no absorber on it. This blue curve shows us that at a given threshold, the printed CD variation is linear according to the defect size. This linearity has not a physical meaning but it depends on the deposition process we modeled. As shown in the part dealing with the impact of a defect on the reflected intensity, we can observe that the CD could not be printed for low defect size. Indeed in this figure, for the threshold we picked, defects with a size below $20 \mathrm{~nm}$ will be not printed. The red curve shows the CD range for a mask structure with an absorber and a gaussian defect. In this study, like in the previous one, The defect lies just underneath the absorber position.

For low defect size, it is the absorber which drive the line width of the printed feature. It is necessary true for non printed defect, but it is still valid for low printable defects (until 30nm in the studied case). On the other hand, when the printed $\mathrm{CD}$ fixed by the defect becomes larger than the $\mathrm{CD}$, then the line width is not driven anymore by the absorber but by the defect. 


\section{CONCLUSIONS}

In this article, the influence of defect in 2D modeling EUV mask has been studied. Different ways to model the defect shape have been investigated, but finally in our simulations, the shape of the defect (circular, elliptic, gaussian, etc...) does not play a significant role. The main parameter is the model chosen to represent the deposition process. Models of planarization effect that we described in the subsection entitled "model of defect shape" do not represent physically the deposition processes, nevertheless, they allow to perform some realistic simulations thanks to the adjustment of the deformation at top of multi-layer mirror. At present, many experiments are carried out to know exactly the defect shape propagation through the layers of the mirror. With these data, it will be possible, in the future, to establish and to manage a precise model of the planarization process. The study of the defective multi-layer with no absorber have shown that the planarization conditions can help in having a low impact of defects. But it has also been demonstrated [11] that a flat topography at top of mirror does not guarantee a defect free printing.

In 2D EUV mask modeling, that is with an absorber layer, the impact of a defect has been investigated. If the defect lies just underneath the absorber, the printed CD will be not disturbed by the defect if the deformation size on surface is smaller than the absorber width. It means that the absorber completely covers the deformation. In this configuration only a low thickness of the absorber allows to point out a defect impact. But this influence is not very dramatic because it will strengthen the drop of intensity caused initially by the absorber layer. The impact of defect is more important if the size of the deformation at the surface is larger than the absorber. In this case, the width of the aerial image is larger than those obtained with a defect free structure and the printed $\mathrm{CD}$ is fixed by the defect and not anymore by the absorber. If the defect is not exactly just below the absorber but still in the vicinity, it will also have a major impact on the CD size. We have shown in this article than the shadowing effect plays a role on the $\mathrm{CD}$ with respect to the position of the defect compared at the absorber position, just on left or just on right. The impact of the shadowing effect can be reduced by adjusting different absorber parameters like the material or the slope angles but also the light incidence. Finally, if the defect is far enough from the absorber, the defect will not disturb the CD printed with the absorber layer and the study of the defect impact is the same than in case of multi-layer with no absorber.

\section{BIBLIOGRAPHY}

[1] P. Evanschitzky, A. Erdmann, M. Besacier, and P. Schiavone: "Simulation of Extreme Ultraviolet Masks with Defective Multilayers", Proc. SPIE Conf. Photomask and Next-Generation Lithography Mask Technology X, 2003 , vol. 5130, p. 1035.

[2] M. Ito, T. Ogawa, K. Otaki, W. Nishyama, S. Okazaki, and T. Terasawa: "Simulation of Multilayer defects in Extreme Ultraviolet Masks" Jpn. J. Appl. Phys. 40 (2001), 2549.

[3] B. S. Bollepalli and F. Cerrina: "Reflection from EUV Masks", Proc. SPIE Conf. Emerging Lithographic Technologies III, Santa Clara (USA), 1999, vol. 3676, p. 587.

[4] M. Brukman, Y. Deng, A. Neureuther : "Simulation of EUV Multilayer Mirror Buried Defects“, Proc. SPIE Conf. Emerging Lithographic Technologies IV, Santa Clara (USA), 2000, vol. 3997, p. 799.

[5] T. Pistor, A. Neureuther:“ Extreme ultraviolet mask defect simulation “, J. Vac. Sci. Technol. B 17 (1999), p 3019.

[6] P. B. Mirkarimi, S. L. Baker, and D. G. Stearns:” Investigating the growth of localized defects in thin films using gold nanospheres", J. Vac. Sci. Technol. B 19 (2001).

[7] E.Quesnel, J.Hue, V.Muffato, C.Pellé and P.Lamy, "Investigation of nodule growth in IBS Mo/Si multilayers for EUV lithography masks”, J. of Vac. Sci. Technol. B 22(5), Sep/Oct 2004.

[8] G.F. Cardinale, A.K. Ray-Chaudhuri, A. Fisher, P.S.J. Mangat, J. Wasson, P.B. Mirkarimi, E. Gullikson:” Effects of smoothing on defect printability at extreme ultraviolet wavelengths", J. Vac. Sci. Technol. B 18(6) (Nov/Dec 2000), p 3019.

[9] V. Farys, P. Schiavone, F. Polack, C. Vannuffel, E. Quesnel, and M. Bertolo: Proc. 2nd International EUV Lithography Symposium, Antwerp, 2003. 
[10] P. Naulleau, K.A. Goldberg, E.H. Anderson:“ Lithographic characterisation of the printability of programmed extreme ultraviolet substrate defects”, J. Vac. Sci. Technol. B 21(4) (Jul/Aug 2003), p 1286.

[11] P.Schiavone, R.Payerne, "Rigorous simulation of line defects in EUV mask", Microprocesses and Nanotechnology Conference, MNC 2001, Matsue, Japan, 2001.

[12] X. Lee: http://www.xahlee.org/SpecialPlaneCurves_dir/Parallel_dir/parallel.html.

[13] T.Pistor, Y. Deng, A. Neureuther:” Extreme ultraviolet mask defect simulation: Low profile defects“, J. Vac. Sci. Technol., B 18(6), pp 2926, Nov/Dec (2000).

[14] M. Besacier, P. Schiavone:" Shadowing effect minimization in EUV mask by modelling", Photomask and NextGeneration Lithography Mask Technology XI, Yokohama, Japan, 2004, vol 5446. [5446-80].

[15] K.Toh et al. "EUV stepper characterization using lithography modeling", $2^{\text {nd }}$ annual International Workshop on Extreme Ultraviolet Lithography, Oct 16-19, 2000. 